\title{
Transhepatic anterograde biliary drainage using a self-expandable metallic stent: A therapeutic alternative after hepaticojejunostomy with Roux-en-Y reconstruction
}

\author{
Fernando Macías-García, Julio Iglesias-García, José Lariño-Noia and J. Enrique Domínguez-Muñoz
}

Gastroenterology Department. Foundation for Research in Digestive Diseases. Hospital Universitario de Santiago de Compostela. A Coruña, Spain

\begin{abstract}
Endoscopic drainage is the procedure of choice in cases of obstructive jaundice. However, in patients with a surgical biliary reconstruction, this technique cannot be often satisfactorily used. In these cases, the best alternative has usually been the percutaneous biliary drainage. Since the introduction of endoscopic ultrasoundguided therapy, some new techniques have been proposed to solve these technical problems related to postsurgical disturbed anatomy. In this case report, we describe our successful experience in the performance of a transhepatic anterograde biliary drainage using a self-expandable metallic stent in the context of a patient who previously had undergone a hepaticojejunostomy with Roux-en-Y reconstruction because of a resected hilar cholangiocarcinoma.
\end{abstract}

Key words: Obstructive jaundice. Biliary drainage. Transgastric drainage. Endoscopic ultrasound.

\section{INTRODUCTION}

Endoscopic biliary drainage (EBD) may be challenging after the performance of a surgical biliopancreatic reconstruction. Several factors, including postsurgical anatomical changes may influence the success of the endoscopic procedure.

Macías-García F, Iglesias-García J, Lariño-Noia J, DomínguezMuñoz JE. Transhepatic anterograde biliary drainage using a self-expandable metallic stent: A therapeutic alternative after hepaticojejunostomy with Roux-en-Y reconstruction. Rev Esp Enferm Dig 2013;105:363-365.

Received: 26-10-2012

Accepted: $17-01-2013$

Correspondence: Fernando Macías-García. Gastroenterology Department. Hospital Universitario de Santiago de Compostela. C/Choupana, s/n. 15706 Santiago de Compostela. A Coruña, Spain

e-mail: fmacgar@yahoo.es

\section{CASE REPORT}

We report the case of a patient with a previous resected hilar cholangiocarcinoma (Klatskin type IV, according to Bismuth classification) six years ago. After dissection of the hilar plate, a hepaticojejunostomy with Roux-en-Y reconstruction was performed (double anastomosis of the right biliary ducts and another anastomosis to the reconstructed left biliary ducts), followed by PFL (cisplatin, 5-fluorouracil, and leucovorin) adjuvant chemotherapy.

The patient was admitted to our hospital with progressive jaundice, constitutional syndrome, anorexia, and weight loss. Laboratory tests revealed the presence of moderate cholestasis $(8.8 \mathrm{mg} / \mathrm{dL}$ total bilirubin, $6.1 \mathrm{mg} /$ dL direct bilirubin), and cholestasis with 937 UI/L gammaglutamyl-aminotransferase (GGT) and $2271 \mathrm{UI} / \mathrm{L}$ alkaline phosphatase (ALP). Significant increase of Ca 19.9 (454 U/mL) was also observed.

Abdominal CT scan showed a heterogeneous and calcified large mass $(10 \times 9 \mathrm{~cm})$ with delayed contrast-enhancement suggestive of a cholangiocarcinoma recurrence. The lesion was located at the segment VIII of the liver with extension to the nearest liver segments and to the porta hepatis. A significant dilatation of the intrahepatic bile ducts and slight decrease of the flow of right branch of the portal vein were also observed (Figs. $1 \mathrm{~A}$ and B).

Since a previous attempt of percutaneous drainage had failed, and no further surgery was considered, we decided to perform an internal biliary drainage assisted by endoscopic ultrasound (EG-3870UTK, Pentax Europe GmbH, Hamburg, Germany), because of the presence of the previously mentioned postsurgical biliary changes, which hampered the usual approach of the papilla. A single dose of ciprofloxacin ( $400 \mathrm{mg}$ i.v.) was administered before the procedure, as usual before an ERCP in our hospital. From the upper body of the stomach and using a 19-gauge Echotip $^{\circledR}$ needle (Cook Endoscopy Inc, Limerick, Ireland), a dilated left bile-duct branch was punctured using color- 
doppler to avoid vessels (Fig. 2A). After removing the stylet, bile was aspirated to confirm the right position into the bile duct. Contrast was then injected, in order to visualize the ducts under fluoroscopy. A 0.035-inch guidewire (Hydra Jagwire $^{\circledR}$, Boston Scientific, Natick, MA, USA) was then advanced to the jejunum through the surgical hepaticojejunostomy (Fig. 2B). A needle knife (Boston Scientific, Natick, MA, USA) was used to create a fistula between the stomach and the biliary tree. Dilation was performed by using a $6 \mathrm{~mm}$ dilating balloon (Fig. 2C), which was also
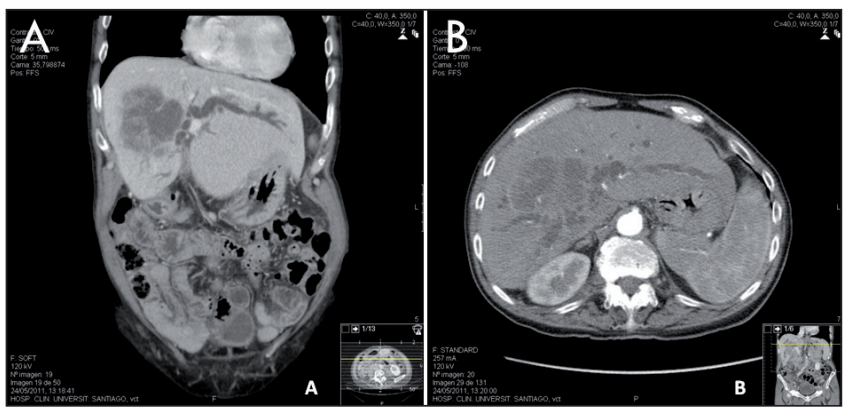

Fig. 1. Abdominal computed tomography images (A. Coronal view and $B$. Transversal view), showing the cholangiocarcinoma recurrence at the segment VIII with extension to the nearest liver segments and to the porta hepatis. Note the significant dilatation of the bilateral intrahepatic bile ducts.
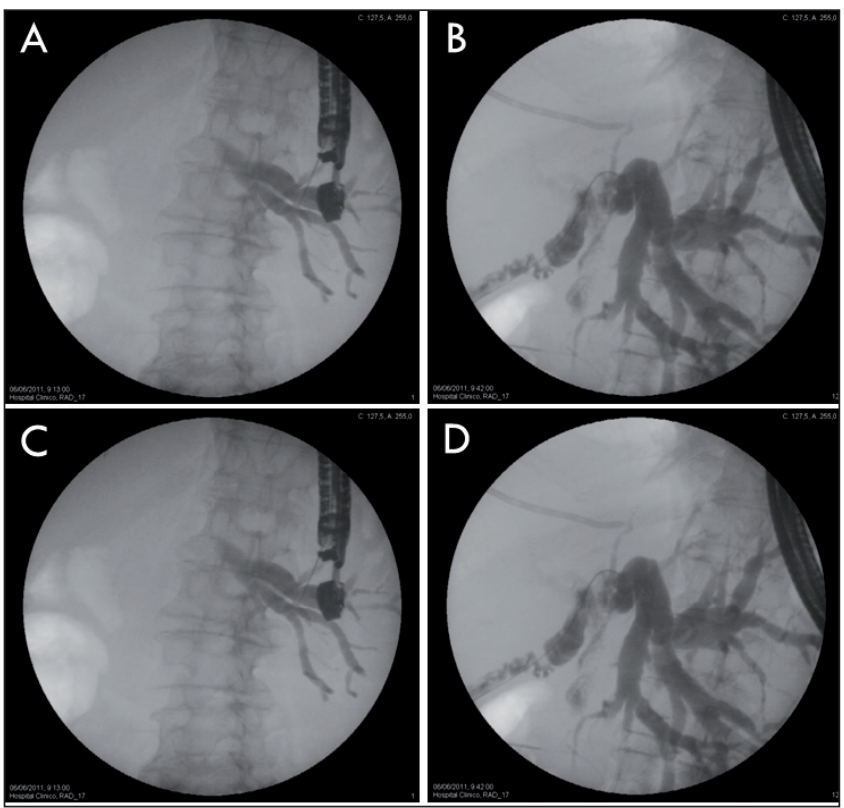

Fig. 2. Fluoroscopic views that show initial steps to access the intrahepatic biliary tree by using a transhepatic anterograde approach. A. Puncture of a dilated peripheral left bile duct guided by endoscopic ultrasonography. B. A 0.035-inch hydrophilic guidewire is advanced to the jejunum through the hepaticojejunostomy. C \& D. A $6 \mathrm{~mm}$ dilatation balloon is employed to dilate the gastric fistula and the intrahepatic biliary tract to the bilioenteric anastomosis. used to dilate the rest of the intrahepatic tract (Fig. 2D) to the bilioenteric anastomosis (Fig. 3A). Then, under fluoroscopic guidance, we proceeded to the transgastric and transhepatic placement of an uncovered self-expandable metallic stent - Wallflex ${ }^{\circledR}(10 \times 60 \mathrm{~mm})$, Boston Scientific, Natick, MA, USA - through the bilioenteric anastomosis, saving completely the tumor stenosis (Fig. 3 B and C). Finally, another plastic stent was placed (7 Fr-10 cm, Boston Scientific, Natick, MA, USA) between the left bile duct and the gastric lumen, in order to avoid bile leakage after the gastric wall dilatation (Fig. 3D). Three weeks later the plastic stent was endoscopically removed allowing the gastric fistula to be closed. No procedure-related complications were observed during the follow-up.

A partial resolution of the obstructive jaundice was observed in the following days, $(4.5 \mathrm{mg} / \mathrm{dL}$ total bilirubin, $3.1 \mathrm{mg} / \mathrm{dL}$ conjugated bilirubin, $457 \mathrm{UI} / \mathrm{L}$ GGT and 960 UI/L ALP), enough for restarting chemotherapy. Despite this, patient died 10 months after the procedure because of tumor progression.

\section{DISCUSSION}

Endoscopic biliary drainage is a widely used technique in the case of obstructive jaundice (1). However, after the completion of a Roux-en-Y hepaticojejunostomy, the endo-
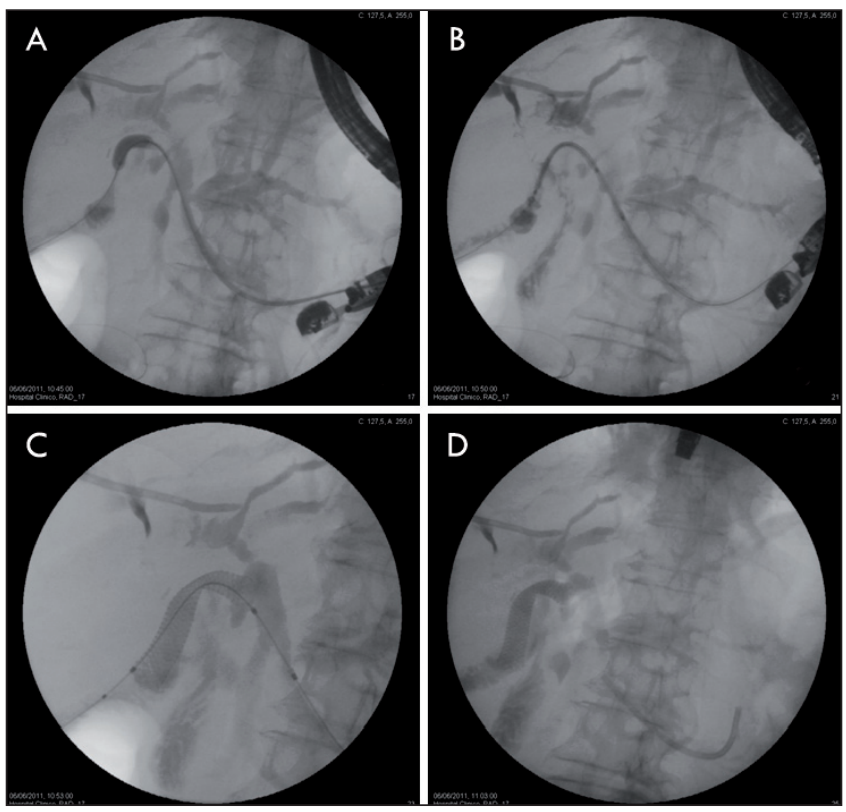

Fig. 3. Fluoroscopic views that show final steps to reach the biliary drainage by using a transhepatic anterograde approach. A. The bilioenteric anastomosis is dilated with a $6 \mathrm{~mm}$ dilatation ballon B. Intrahepatic advance of the uncovered self-expandable metallic stents (SEMS) to the hepaticojejunostomy. C. Hourglass aspect of the SEMS deployed in the stenotic anastomosis. D. Final view of the SEMS at the hepaticojejunostomy and another plastic stent at the gastric wall. 
scopic access to the bilioenteric anastomosis is not always possible, mainly due to the surgically altered anatomy (2). In these cases, percutaneous transhepatic biliary drainage (PTBD) has been the alternative of choice until the appearance of EUS-guided biliary drainage (EUS-BD) (3). The classical technique of percutaneous transhepatic drainage is commonly associated with high morbidity and complication rates (4), ranging from 8 to $30 \%$. The most commonly complications include cholangitis, sepsis, bile leak, pain, and catheter dislocation.

EUS-guided hepaticogastrostomy was first reported by Burmester et al. in 2003 (5). Kahaleh have described the advantages of EUS-guided hepaticogastrostomy over percutaneous transhepatic drainage, including puncture of the biliary tree with real-time US when using color-doppler information to avoid vascular injury, the lack of ascites in the interventional field when present in the peritoneum, and the lack of an external drainage (6). However, limitations of this technique have also been described: non-apposed gastric wall and left liver lobe, with certain displacement between the puncture site of the gastric wall and the intrahepatic bile duct, leading to technical failure; the difficulty of puncture in case of liver cirrhosis; the risk of injuring portal vein and the need of using small-caliber stents or metal stent with small-diameter delivery device (7). Regarding complication rates, in Spanish multicenter study a $23 \%$ complication rate following EUS-guided cholangiopancreatography has been recently reported (8).

Using this transgastric access, Nguyen-Tang first described in 2010 the EUS-guided transhepatic anterograde self-expandable metal stent placement for biliary obstruction, in a single session (9). EUS-guided anterograde SEMS placement has advantages over the other biliary drainage modalities, as the elimination of the probability of skin infection and percutaneous technical limitations caused by obesity or the presence of ascites. Bile leak or stent dislodgement potentially associated with the EUS-guided transmural approach (hepaticogastrostomy or choledochoduodenostomy), are also prevented by leaving the SEMS entirely intrahepatic. In our case we preferred to prevent the bile leakage by the temporary placement of a transgastric plastic stent.

In conclusion, transhepatic anterograde self-expandable metal stent placement guided by EUS, for biliary obstruction seems to be a safe alternative to achieve an adequate biliary drainage in selected patients. Prospective studies to confirm feasibility and safety are needed.

\section{REFERENCES}

1. Giovannini M, Bories E. EUS-Guided Biliary Drainage. Gastroenterol Res Pract 2012;2012:348719.

2. Artifon EL, Safatle-Ribeiro AV, Ferreira FC, Poli-de-Figueiredo L, Rasslan S, Carnevale F, et al. EUS-guided antegrade transhepatic placement of a self-expandable metal stent in hepatico-jejunal anastomosis. JOP 2011;12:610-3.

3. Yamao K, Hara K, Mizuno N, Sawaki A, Hijioka S, Niwa Y, et al. EUSGuided Biliary Drainage. Gut Liver 2010;4(Supl. 1):S67-75.

4. Stoker J, Lameris JS. Complications of percutaneously inserted biliary wallstents. J Vasc Interv Radiol 1993;4:767-72.

5. Burmester E, Niehaus J, Leineweber T, Huetteroth T. EUS-cholangio-drainage of the bile duct: Report of 4 cases. Gastrointest Endosc 2003;57:246-51.

6. Kahaleh M, Hernandez AJ, Tokar J, Adams RB, Shami VM, Yeaton P. Interventional EUS-guided cholangiography: Evaluation of a technique in evolution. Gastrointest Endosc 2006;64:52-9.

7. Itoi T, Sofuni A, Itokawa F, Tsuchiya T, Kurihara T, Ishii K, et al. Endoscopic ultrasonography-guided biliary drainage. J Hepatobiliary Pancreat Sci 2010;17:611-6.

8. Vila JJ, Pérez-Miranda M, Vázquez-Sequeiros E, Abadia MA, PérezMillán A, González-Huix F, et al. Initial experience with EUS-guided cholangiopancreatography for biliary and pancreatic duct drainage: a Spanish national survey. Gastrointest Endosc 2012;76:1133-41.

9. Nguyen-Tang T, Binmoeller KF, Sanchez-Yague A, Shah JN. Endoscopic ultrasound (EUS)-guided transhepatic anterograde self expandable metal stent (SEMS) placement across malignant biliary obstruction. Endoscopy 2010;42:232-6. 